\title{
Telocytes role during the postnatal development of the Mongolian gerbil jejunum
}

\author{
Bruno C. Zani ${ }^{\mathrm{a}}$, Bruno D.A. Sanches ${ }^{\mathrm{b}}$, Juliana S. Maldarine ${ }^{\mathrm{b}}$, Manoel F. Biancardi ${ }^{\mathrm{c}}$, \\ Fernanda C.A. Santos ${ }^{c}$, Caroline N. Barquilha ${ }^{\mathrm{d}}$, Mariele I. Zucão ${ }^{\mathrm{a}}$, Carolina M.B. Baraldi ${ }^{\mathrm{a}}$, \\ Sergio L. Felisbino ${ }^{\mathrm{a}, \mathrm{d}}$, Rejane M. Góes ${ }^{\mathrm{a}, \mathrm{b}}$, Patricia S.L. Vilamaior ${ }^{\mathrm{a}}$, Sebastião R. Taboga ${ }^{\mathrm{a}, \mathrm{b}, *}$ \\ ${ }^{a}$ Univ. Estadual Paulista - UNESP, Department of Biology, Laboratory of Microscopy and Microanalysis, Cristóvão Colombo St., 2265, São José do Rio Preto, São Paulo, \\ Brazil \\ ${ }^{\mathbf{b}}$ Department of Structural and Functional Biology, State University of Campinas, Bertrand Russel Av., Campinas, São Paulo, Brazil \\ ${ }^{\mathrm{c}}$ Department of Histology, Embryology and Cell Biology, Federal University of Goiás, Samambaia II, Goiânia, Goiás 74001970, Brazil \\ d Univ. Estadual Paulista - UNESP, Institute of Biosciences, Prof. Dr. Antônio Celso Wagner Zanin St., 250, Rubião Júnior District, Botucatu, São Paulo 18618-689, Brazil
}

\section{A R T I C L E I N F O}

\section{Keywords:}

Telocytes

CD34

Muscularis mucosae

C-kit

Differentiation

Weaning

\begin{abstract}
A B S T R A C T
Telocytes are recently categorised CD34-positive interstitial cells that comprise the cells which were previously called interstitial Cajal-like cells (ICLCs). These were detected in the stroma of various organs such as the prostate, lungs, mammary glands, liver, gallbladder, and jejunum, among others. Several functions have been proposed for telocytes, such as a supportive role in smooth muscle contraction and immune function in adult organs, and tissue organisation and paracrine signalling during development, as well as others. In the jejunum, little is known about the function of telocytes in the adult organ, or is there any information about when these cells develop or if they could have an auxiliary role in the development of the jejunum. The present study employed histological, immunohistochemical and immunofluorescence techniques on histological sections of the jejunum of Mongolian gerbil pups on two different days of postnatal development of the jejunum, covering the maturation period of the organ. By immunolabelling for CD34, it was observed that telocytes are already present in the jejunum during the first week of postnatal life and exist in close association with the developing muscularis mucosae, which are therefore TGFß1-positive. The telocytes are still present at the end of the first month of life, and a portion of them present co-localisation with c-Kit. Fibroblast-like cells, which are exclusively c-Kitpositive, are also observed, which may indicate the presence of interstitial Cajal cells (ICCs). Finally, it can be hypothesised that a portion of the telocytes may give rise to ICCs, which are c-Kit-positive but CD34 negative.
\end{abstract}

\section{Introduction}

Telocytes are newly described cells that are located in the interstices of various organs. These include cells which were previously called interstitial Cajal-like cells (ICLCs) that have been described in several organs (Popescu and Faussone-Pellegrini, 2010), whereas the interstitial cells of Cajal (ICCs) are exclusive to the gastrointestinal tract and were discovered by Ramón y Cajal (1911), so were assumed to be interstitial neurons. Later, it was found that ICCs originate from the mesoderm and a pacemaker role in smooth muscle contraction in the intestine was proposed for them (Thuneberg, 1982). ICCs have c-Kit as the main marker (Komuro and Zhou 1996; Pasternak et al., 2016; Aleksandrovych et al. 2017), which is a receptor that binds to stem cell factor (SCF) and induces calcium mobilization through a kinase of the src family and phosphoinositide 3-kinase (PI3K) (Liang et al., 2013). This receptor would be central to the pacemaker function exerted by the ICCs (Drumm et al. 2014). Telocytes have CD34 as the main marker and a portion of them are also c-kit-positive (Cretoiu et al. 2012); before the establishment of the term telocytes, CD34-positive ICLCs that did not show c-Kit labelling were detected in the intestine (Iino and Nojyo 2009). These cells correspond to that which was later conceptualised as intestinal telocytes (Cretoiu et al. 2012).

Telocytes are morphologically distinct from fibroblasts, with long cytoplasmic projections called telopodes, which in turn have a monoliform aspect, with alternation between dilated regions, podoms (which carry mitochondria, endoplasmic reticulum and cavelola) and fibrillar-

\footnotetext{
* Corresponding author at: Department of Biology, Laboratory of Microscopy and Microanalysis, São Paulo State University, 2265, Cristóvão Colombo Street, Jardim Nazareth, São José do Rio Preto, São Paulo 15054-000, Brazil.

E-mail address: taboga@ibilce.unesp.br (S.R. Taboga).
} 

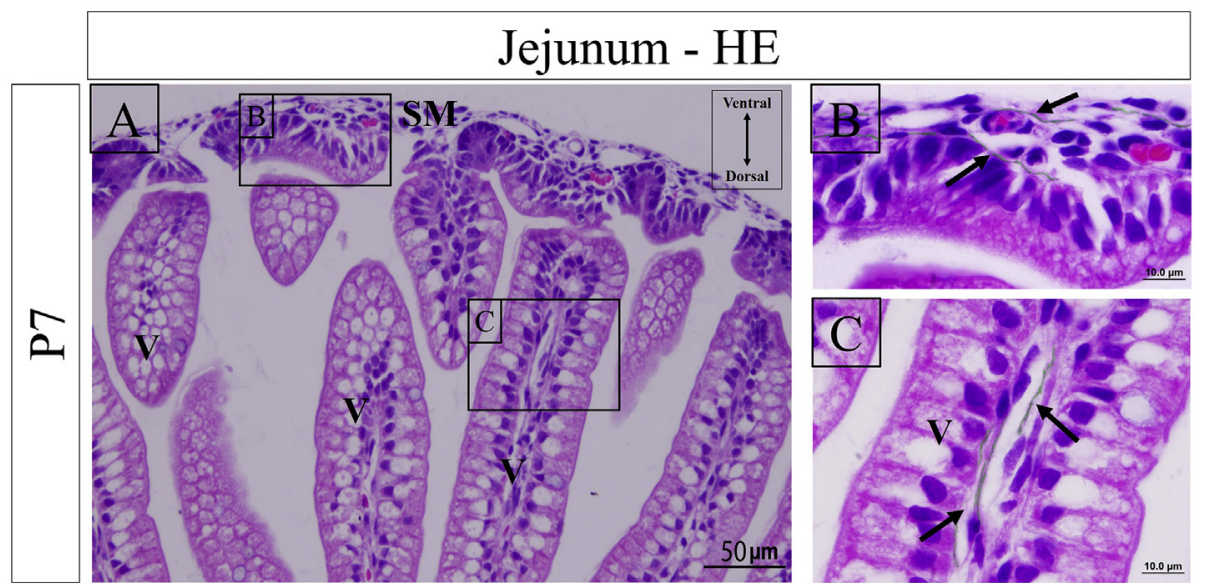
junum stained with HE. (A) Jejunum in development on P7. (B) Telocytes presenting their visible morphological characteristics in light microscopy (long and thin cytoplasmic projections and cylindrical cell bodies) can be observed in the submucosa. (C) Telocytes are also observed in the centre of the villi around the lacteals. (D) Jejunum in development on P30. (E, F) Telocytes are also present at the periphery of the muscularis externa. SM (Submucosa), V (Villi), Arrows (Telocytes), CL (Crypts of Lieberkühn), ME (Muscularis Exerna).
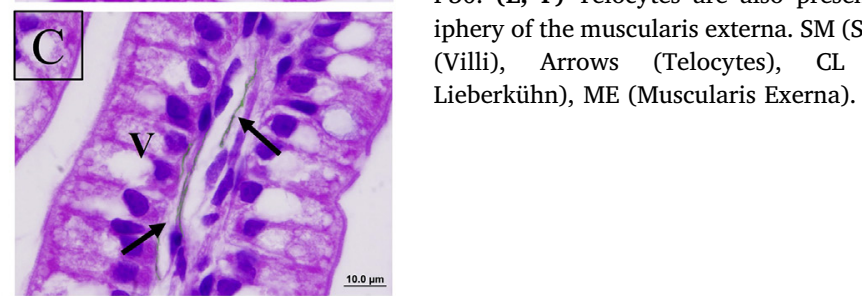
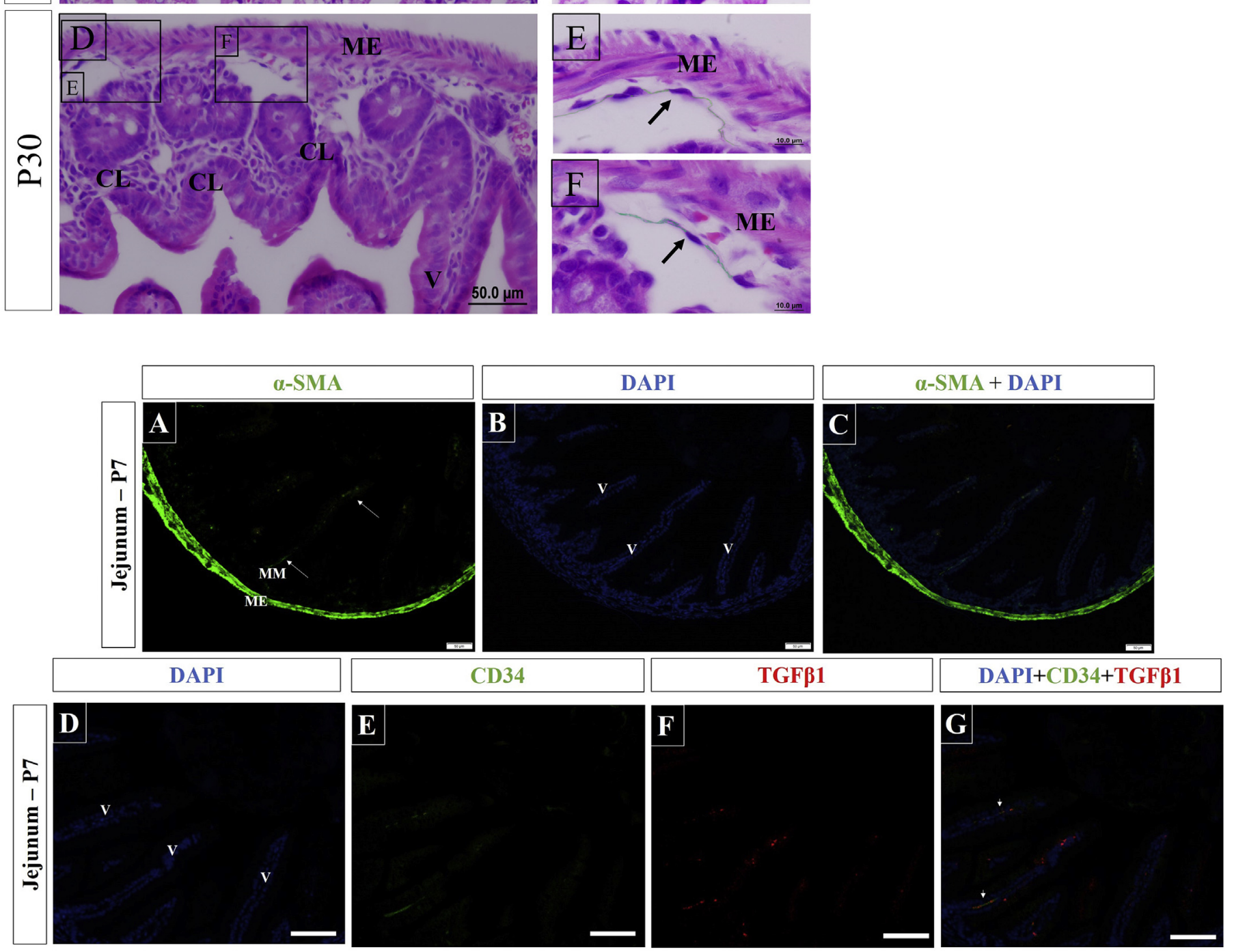

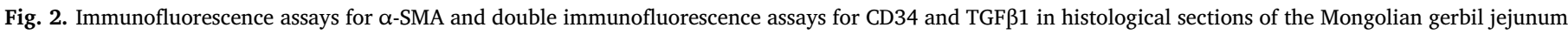

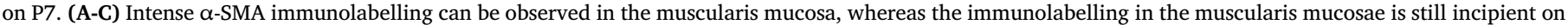

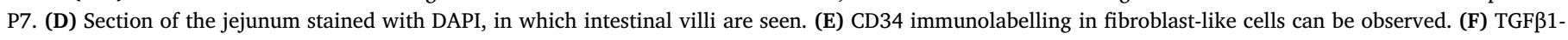

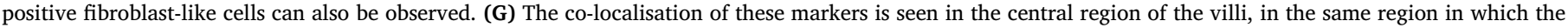

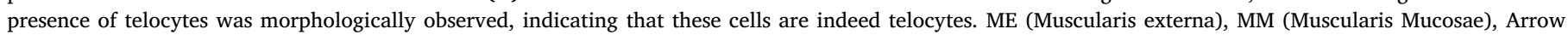
(Smooth muscle cells inside the villi), V (Intestinal villi), Arrowhead (CD34 and TGF $\beta 1$ co-localisation region), Bar (20 $\mu$ ).

like regions called podomers (Popescu and Faussone-Pellegrini, 2010). There is evidence that telocytes differ from fibroblasts in terms of the proteome, morphology and gene expression (Kang et al. 2015; Xiao and Bei, 2016; Zhang et al., 2006) and from ICCs, which have a pyramidal cell body and shorter cytoplasmic projections (Popescu and FaussonePellegrini, 2010). Telocytes have characteristics of progenitor cells, as proposed for a wide subset of CD34-positive cells (Sidney et al., 2014); it was hypothesised that these cells could give rise to fibroblasts and 


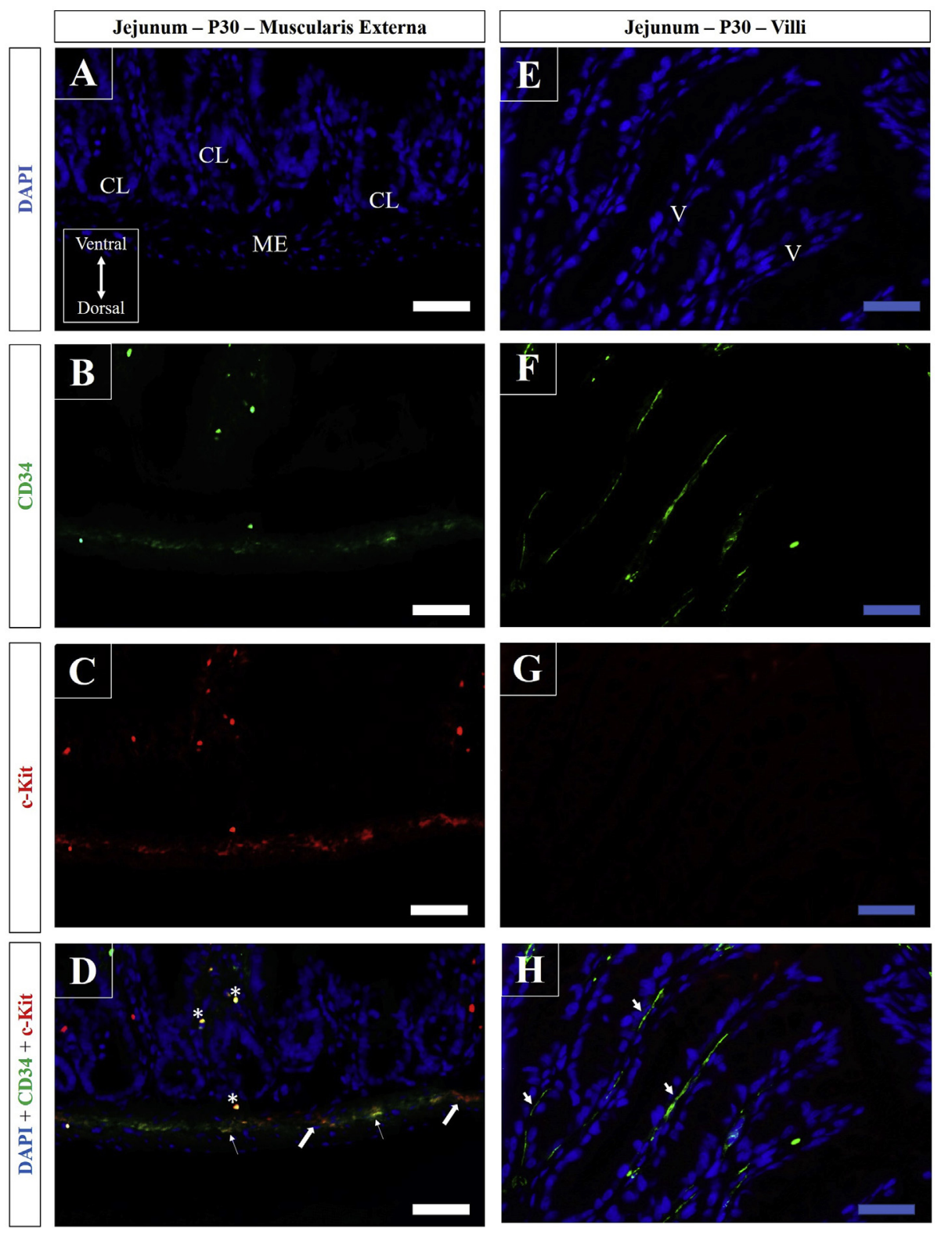

Fig. 3. Immunofluorescence assays for $\alpha$-SMA in sections of the Mongolian gerbil jejunum on P30. (A) At the end of the first month of postnatal life, it is possible to observe the expansion of $\alpha$-SMA immunolabelling, which indicates the expansion of the muscularis mucosae. It is also possible to discriminate the $\alpha$-SMA immunolabelling in the muscularis mucosae; it becomes defined, which indicates the development of this smooth muscle. (B) Muscularis mucosae is observed in detail and its two constituent components, the outer longitudinal smooth muscle and the inner circular smooth muscle, can be discriminated. (C) Emphasis is given to muscularis mucosae in which such muscle can be observed inside the plicae circularis; also, the expansion of it into the villi is seen. (OLM) (Outer longitudinal smooth muscle), ICM (Inner circular smooth muscle), smaller bar $(100 \mu \mathrm{m})$, larger bar $(50 \mu \mathrm{m})$.

myofibroblasts, playing an active role in tissue repair in the skin (DíazFlores et al. 2016), as well as to cardiomyocytes (Bei et al. 2015) or even ICCs (Sanches et al., 2017a, b). In addition, telocytes undergo the intense synthesis of paracrine factors in their vesicles, which would also indicate possible roles in stromal development, as well as the expression of proteins associated with homeostasis and stromal repair (Zheng et al., 2014).

Such cells were described in the liver (Xiao et al., 2013), gallbladder (Pasternak et al. 2012, 2013a-b; Matyja et al. 2013), lungs (Zheng et al., 2012) skin (Ceafalan et al. In the panties (Nicolescu and Popescu, 2012), mammary glands (Mou et al., 2013), parotid glands (Nicolescu et al., 2011), kidneys (Qi et al., 2012), uterus (Creţoiu et al. 2012), prostate (Corradi et al. 2013), duodenum (Carmona et al. 2011) and jejunum (Cretoiu et al. 2012). In the jejunum, a role in intercellular signalling has been hypothesised, such as a role in the control of local organ homeostasis in the duodenum; however, the function of these cells in the intestine is still elusive, and there is no information about the presence of these cells throughout intestinal development. The development of the jejunum of rats is a complex process, which begins in the foetal life. At birth, the jejunum, like the rest of the intestine, is not yet fully mature. From birth to weaning, the rat intestine continues to develop, with an increase in proliferative activity, in villi width and in immunological activity (Cummins et al. 1988; Montgomery et al., 1999, Sureda et al., 2017). The complete network of cell interactions and molecular pathways related to the jejunum development has not been completely elucidated; thus, the presence of telocytes during intestinal development could indicate a greater complexity to this process. Telocytes were also assumed to play a role in the development of the myocardium (Faussone-Pellegrini and Bani, 2010; Bani, 2016) and the prostate (Sanches et al., 2016), especially in terms of tissue organisation and smooth muscle development.

The Mongolian gerbil is a rodent species that is easy to handle in the laboratory, making it promising for experimental manipulation (Kuehn and Zucker, 1968; Norris and Adams, 1972; Spitzer and Semple, 1995). Several experiments ranging from studies on the nervous system, diabetes, and cancer to reproductive biology investigations were performed with gerbils, due to the previously mentioned factors, their hormonal peculiarities and their high susceptibility to epilepsy and tumorigenesis (Loskota et al., 1974; Spitzer and Semple, 1995; Santos and Taboga, 2006, Taboga, Vilamaior and Góes, 2009, Salyards et al., 2013, Sanches et al., 2017b, Li et al. 2016). The gerbil has a gestation period of about 25 days, which is longer than that of mice, which lasts 

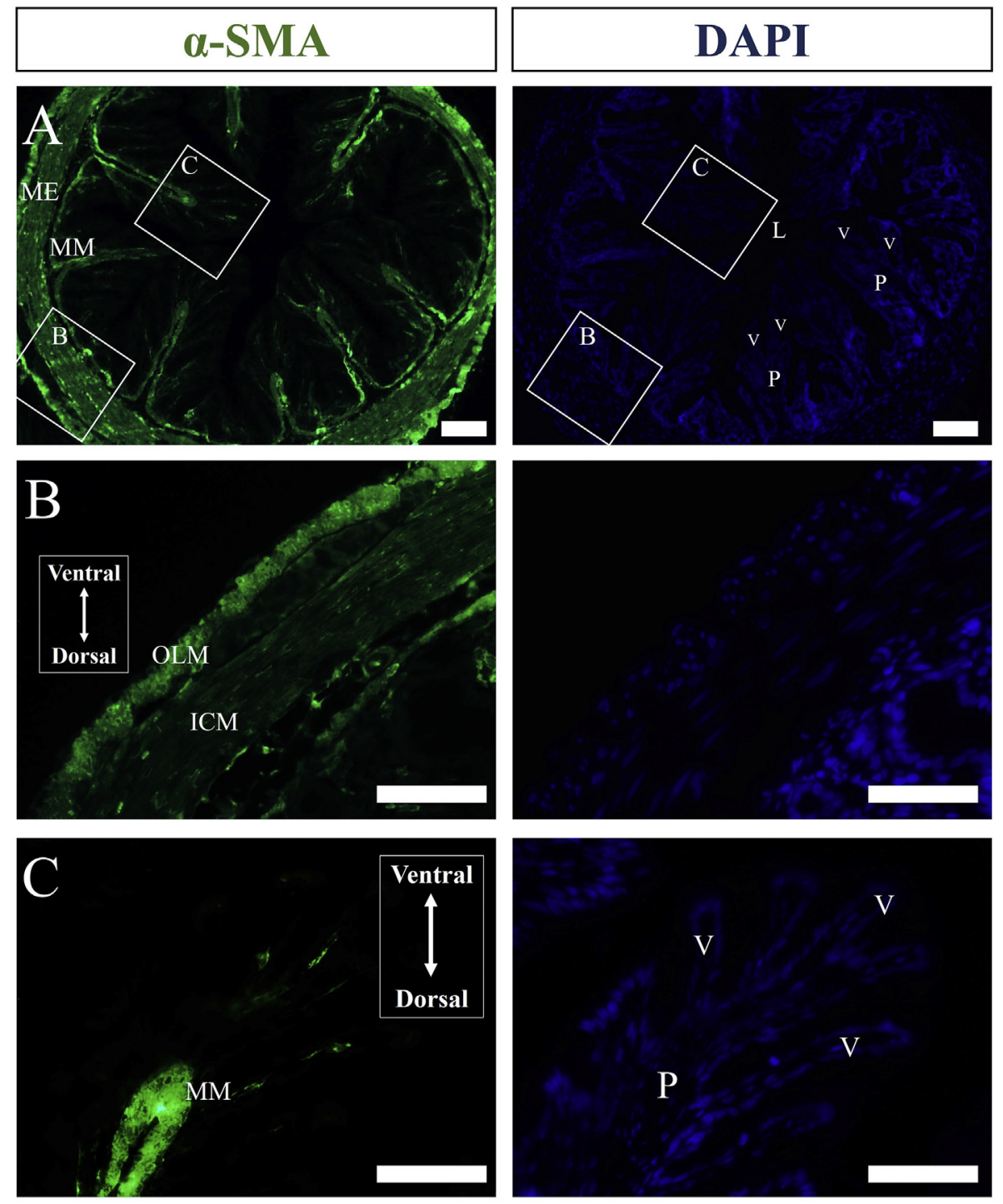
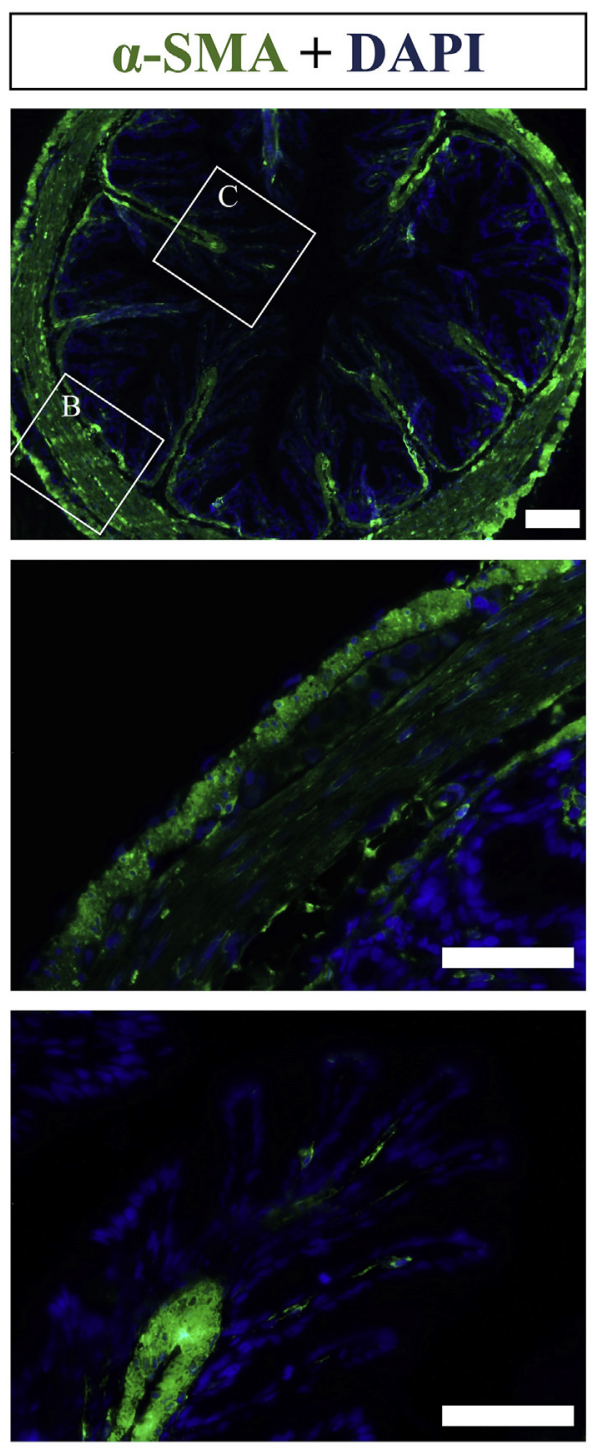

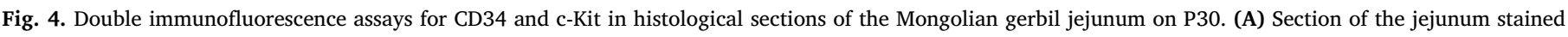

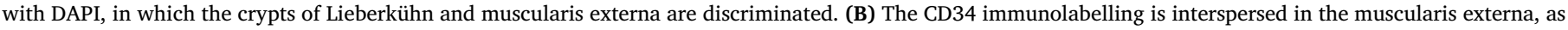

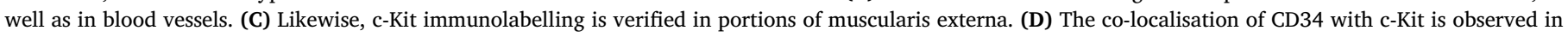

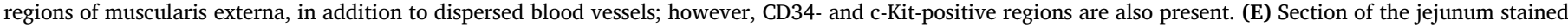

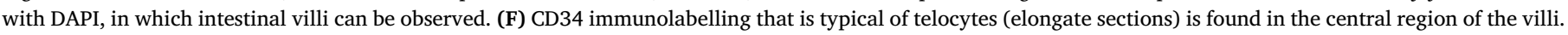

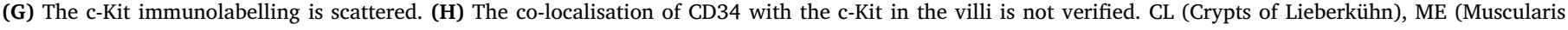

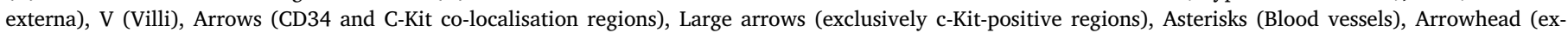

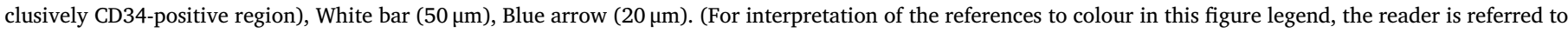
the web version of this article.)

for about 19 days (Sugimura et al., 1986), and that of rats, which lasts for approximately 21 days (Hayashi et al. 1991). Thus, the Mongolian gerbil tends to show a more pronounced postnatal development (Sanches et al., 2014). The weaning period also occurs later, at the end of the first postnatal month (Norris and Adams, 1972), while in the rat, it occurs at the end of the third week (Cummins et al. 1988); therefore, the gerbil becomes useful for studies of the development of the intestine, considering that part of the intestinal development occurs during the postnatal period until the onset of weaning (Sureda et al., 2017). The current study aims to evaluate the presence of telocytes in two distinct periods of the postnatal development of the jejunum in the Mongolian gerbil, as well as to investigate a possible implication of these cells in it.

\section{Material and methods}

\subsection{Animals and experimental design}

The animals were provided by the São Paulo State University (UNESP, São José do Rio Preto). Gerbils were housed in a temperaturecontrolled $\left(25^{\circ} \mathrm{C}\right)$ room on a $12 \mathrm{~h}$ light/dark cycle. All of the animals were housed in polyethylene cages, with ad libitum access to filtered water and rodent food. Animal handling and experiments were performed according to the ethical guidelines of the São Paulo State University (UNESP, Ethical Committee number 115/2015 CEUA), following the Guide for the Care and Use of Laboratory Animals. We used 10 adult female and 10 adult male (between 3 and 4 months old) gerbils (M. unguiculatus, Muridae: Gerbillinae) for mating. We randomly matched one male and one female to form independent litters. After birth, the pups were assigned to form two groups, each containing five male 

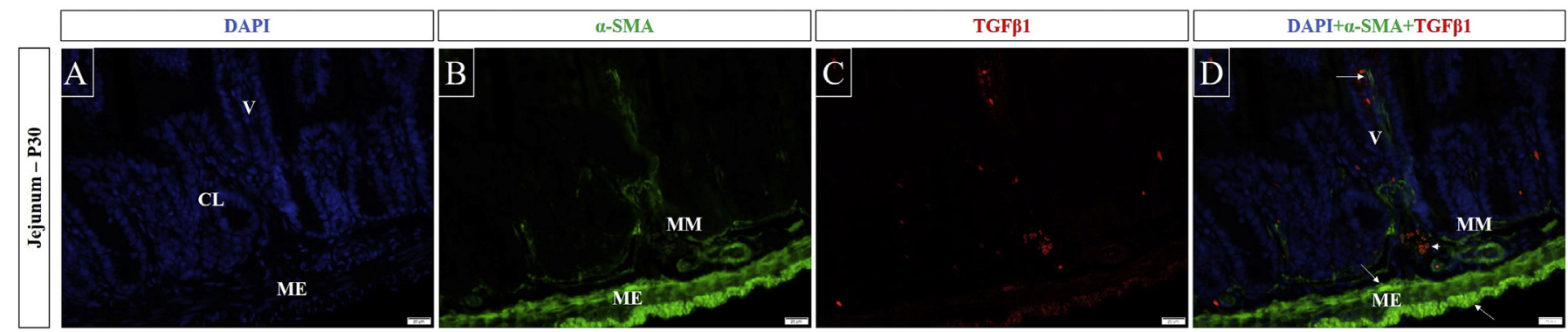

Fig. 5. Double immunofluorescence assays for $\alpha$-SMA and TGF $\beta 1$ in histological sections of the Mongolian gerbil jejunum on P30. (A) Section of the jejunum stained with DAPI, in which the intestinal villi, crypts of Lieberkühn and muscularis externa are present. (B) The $\alpha$-SMA immunolabelling can be verified in the muscularis externa as well as in the muscularis mucosae. (C) TGF $\beta 1$ immunolabelling can be verified in portions of muscularis externa, as well as in vessels and in portions of the villi, coinciding with parts of muscularis mucosae. (D) The co-localisation of TGF $\beta 1$ and $\alpha$-SMA is observed in regions of the muscularis externa, as well as in regions of the muscularis mucosae; there is exclusive TGF $\beta 1$ immunolabelling in the vicinity of the smooth musculature inside the villi, as well as in blood vessels and haematopoietic cells. V (Villi), CL (Crypts of Lieberkühn), ME (Muscularis externa), MM (Muscularis mucosae), Arrow (co-localisation of $\alpha$-SMA with TGFß1), Arrowhead (exclusively TGF 31 -positive region).

pups, to cover the postnatal development of the jejunum in the gerbil: P7 (postnatal day 7) and P30 (postnatal day 30); these groups were used for morphological analysis, as well as immunohistochemistry and immunofluorescence assays. The animals were sacrificed by lethal injection containing a mixture of an anaesthetic, ketamine $(100 \mathrm{mg} / \mathrm{kg}$ bw, Dopalen, Vetbrands, Brazil), and a muscle relaxant, xylazine (11 mg/kg bw, Rompun, Bayer, Brazil).

\subsection{Histological processing}

After dissection, the pups' jejunums were collected and fixed in $4 \%$ buffered paraformol or Karnovsky ( $5 \%$ paraformaldehyde solution and $2.5 \%$ glutaraldehyde in Sörensen pH 7.2 0.1 M phosphate buffer), washed in water, dehydrated in ethanol, clarified in xylol, and then embedded in Paraplast (Histosec, Merk). The organs were sectioned at 3-5 $\mu \mathrm{m}$ and histological slides were mounted; a portion of them was stained by the Haematoxylin-Eosin (HE) histochemical technique for general morphological studies.

\subsection{Immunofluorescence assays}

Immunofluorescences of paraffin-embedded tissues sections were performed using the protocol described in Lima et al. (2015). The jejunos were fixed by immersion in $4 \%$ paraformaldehyde (buffered in $0.1 \mathrm{M}$ phosphate, $\mathrm{pH} 7.4$ ) for $24 \mathrm{~h}$. After fixation, the tissues were washed in water, dehydrated in ethanol series, paraffin embedded (Histosec, Merck, Darmstadt, Germany) and sectioned at $5 \mu \mathrm{m}$ on a Leica microtome (Leica RM2155, Nussloch, Germany). Such sections of jejunum of gerbil pups on the 7th postnatal day of life (P7) and the 30th (P30) were subjected to immunofluorescence assays to evaluate the presence of telocytes throughout the development of the jejunum as a possible function for these cells. Therefore, immunofluorescence assays for a-SMA (rabbit polyclonal, IgG, I-19, sc-1616, Santa Cruz Biotechnology), and double-immunofluorescence assays for CD34 (mouse polyclonal, IgG, B-6, sc74499, Santa Cruz Biotechnology) and c-Kit (rabbit polyclonal, IgG, C-19, sc168, Santa Cruz Biotechnology, CA, USA) and for CD34 (mouse polyclonal, IgG, B-6, sc74499, Santa Cruz Biotechnology) and TGF $\beta 1$ (rabbit polyclonal, IgG, V, sc146, Santa Cruz Biotechnology, CA, USA) were performed; these antibodies were incubated at a dilution of 1:100 overnight. On the following morning, the sections were incubated with goat anti-mouse antibody (FITC, sc-2011, Santa Cruz Biotechnology) and goat anti-rabbit and (sc-2780, Santa Cruz Biotechnology, USA - Texas Red) secondary antibodies diluted 1:200 in 1\% BSA for $2 \mathrm{~h}$ at room temperature and then stained with DAPI (F36924, Life Technology, Grand Island, NY, USA). Histological sections were analysed with a ZeissImager M2 fluorescence microscope (Zeiss, Germany) coupled with AxioVision software (Zeiss, Germany).

\subsection{Immunohistochemistry assays}

The jejunum sections of pups on P7 and on P30 were submitted to immunohistochemistry for PCNA detection using the protocol adopted in Fochi et al. 2013). Antibodies (at a dilution of 1:100) were used for the detection of PCNA (monoclonal mouse IgG2a, PC10, sc-56, Santa Cruz Biotechnology). PolyK4061 K4061 (DAKO Envision DAKO, North America, Carpinteria, USA) was used as the secondary antibody. The incubation time was $45 \mathrm{~min}$ at $37^{\circ} \mathrm{C}$. Later, the assays were performed through the use of diaminobenzidine (DAB) and the sections were counterstained with Harris haematoxylin. Histological sections were analysed with the Olympus BX60 (Olympus) light microscope. Negative controls were obtained by omission of the step of incubating the primary antibody. The assays for were quantified according to the methodology described below.

\subsection{Quantification of immunohistochemistry assays}

For the quantification immunohistochemical assays for PCNA, which were performed to evaluate the proliferative profile of different jejunum compartments between P7 and P30, the total number of cells in 30 fields was counted at random (at $20 \times$ magnification) for each group derived from 3 different animals; this was according to the methodology described in Fochi et al. (2013), in order to separate the cells into positively- and negatively-labelled. A minimum of 1000 cells was counted per group and then the percentage of immunostaining was calculated as the number of positive cells divided by the total number of cells. Quantitative results are expressed as mean \pm standard error (SD).

\subsection{Statistics analysis}

The data were initially studied by Analysis of Variance (One-way ANOVA) and, subsequently, by the Tukey Test for multiple comparisons, with a significance level of $5 \%(p \leq .05)$. The statistical tests were performed with Statistica 7.0 software (StarSoft Inc., Tulsa, OK, USA).

\section{Results}

\subsection{Histology}

In the histological sections stained by HE in the developing jejunum on P7 (Fig. 1A), telocytes can be seen, presenting morphological characteristics that are visible in light microscopy (long and thin cytoplasmic projections and cylindrical cell bodies) in the submucosa (Fig. 1B) as well as in the centre of villi around the lacteals (Fig. 1C). In P30, telocytes are verified in the external muscularis (Fig. 1D-F). 


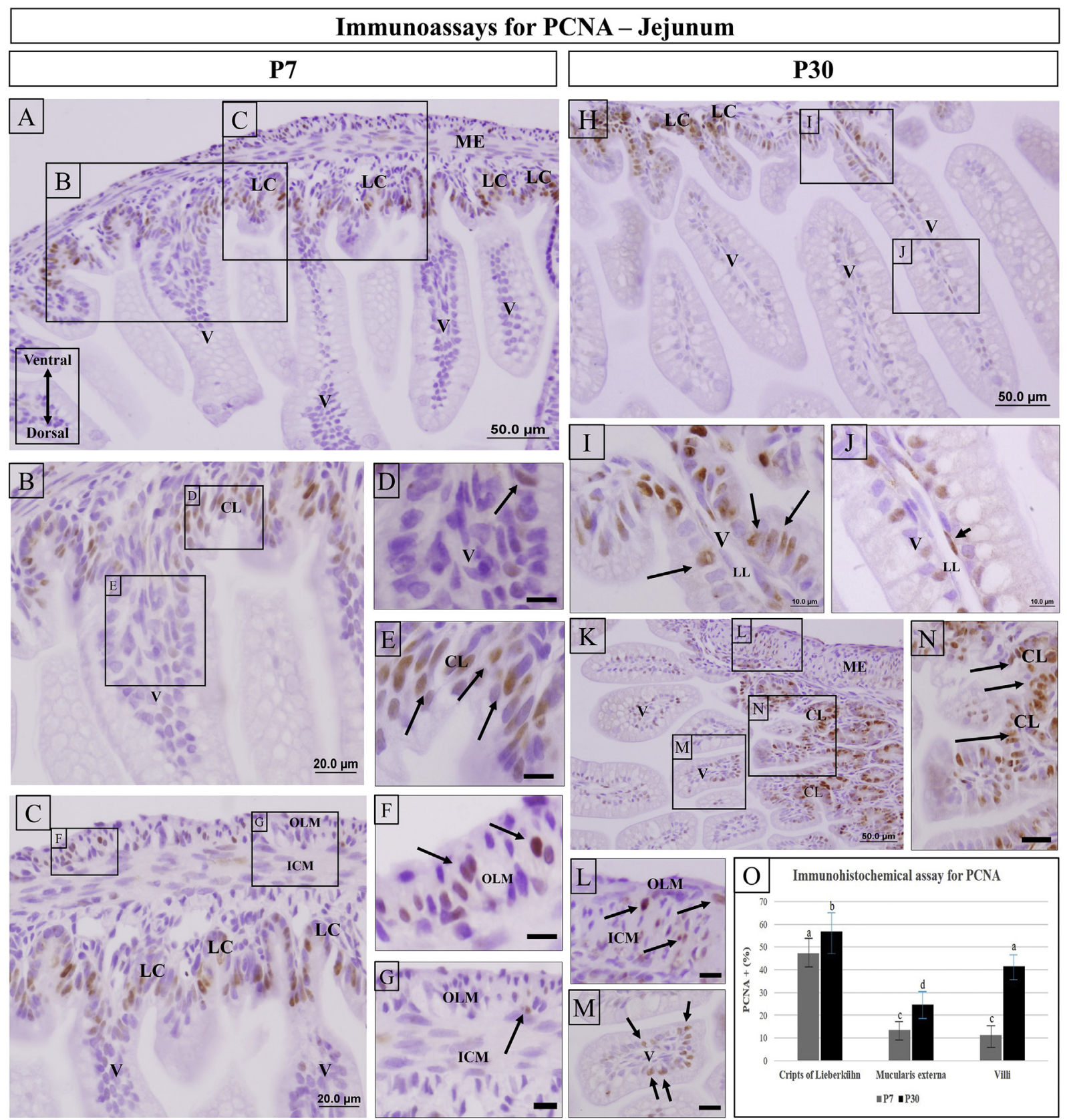

Fig. 6. Immunohistochemical assays for PCNA in sections of the Mongolian gerbil jejunum on P7 and P30. (A) Section of the jejunum of the Mongolian gerbil on P7, in which villi, muscularis externa and the crypts of Lieberkühn are observed. (B) Detail of the jejunum in which emphasis is given to the villi and the crypts of Lieberkühn. (C) Detail of the jejunum in which the muscularis externa is emphasised. (D) Detail of the villus in which scattered PCNA immunolabelling is seen, indicating low proliferative activity. (E) Detail of a Lieberkühn crypt in which there is a large number of PCNA-positive cells. (F) Detail of the outer longitudinal smooth muscle, in which a large number of PCNA-positive cells is verified. (G) Detail of inner circular smooth muscle, in which scattered immunolabelling is verified. (H) Section of the jejunum on P30, in which villi and crypts of Lieberkühn are seen. (I) Detail of the villi, in which a large number of PCNA immunolabelled cells are verified. (J) Detail of a villus, in which a possible telocyte labelled for PCNA can be observed. (K) Detail of the jejunum, in which the muscularis externa can be observed, as well as the crypts of Lieberkühn and villi. (L) Detail of muscularis externa, in which a large number of PCNA-positive cells can be observed. (M) Detail of a villus, in which a large number of PCNA-positive cells are seen. (N) Detail of Lieberkühn crypts, in which intense PCNA immunolabelling is verified. (O) Graph of PCNA immunoassay quantification, an increase in the percentage of cells marked for PCNA between P7 and P30 is seen in the Lieberkühn crypts, in the muscularis externa, and in the villi. Of the three compartments analysed, the highest increase was observed in the villi. V (Villi), CL (Crypts of Lieberkühn), ME (Muscularis externa), OLM (Outer longitudinal smooth muscle), ICM (Inner circular smooth muscle), black bar $(10 \mu \mathrm{m})$.

\section{2. $\alpha$-SMA assays on P7}

On P7, intense labelling for $\alpha$-SMA can be observed in the muscularis externa, although the labelling on the muscularis mucosae is still incipient (Fig. 2A-C). CD34 immunolabelling in fibroblast-like cells can be seen in the villi. TGF $\beta 1$ immunolabelling on fibroblast-like cells can also be observed (Fig. 2D-F). The co-localisation of these markers is observed in the central region of the villi, in the same region where the presence of telocytes was morphologically verified, which corroborates the presence of these cells in the developing villi (Fig. 2G).

\section{3. $\alpha$-SMA assays on P30}

The expansion of $\alpha$-SMA immunolabelling is verified in the jejunum 
on P30, which indicates in turn the expansion of the muscularis externa; it is also possible to discriminate the labelling for $\alpha$-SMA in the muscularis mucosae, which becomes well-defined, indicating the development of this smooth muscle (Fig. 3A). In the muscularis externa, its two constituent components can be observed, the outer longitudinal smooth muscle and the inner circular smooth muscle (Fig. 3B). As for muscularis mucosae, it can be observed that the musculature extends inside the plicae circularis, reaching the interior of the villi (Fig. 3C).

\subsection{Double immunofluorescence assays for CD34 and c-Kit on P3O}

CD34 immunolabelling is interspersed in the muscularis externa, as well as in blood vessels. Likewise, c-Kit immunolabelling is verified in portions of the muscularis externa (Fig. 4A-C). The co-localisation of CD34 and c-Kit is observed in portions of the muscularis externa, in addition to blood vessels; however, regions that are exclusively positive for CD34 and c-Kit can be verified (Fig. 4D). CD34 immunolabelling typical of telocytes (elongated sections) is seen in the central region of the villi, whereas the immunolabelling for c-Kit appears to be dispersed (Fig. 4E-G). The co-localisation of CD34 with c-Kit in the villi is not verified (Fig. 4H).

\subsection{Double immunofluorescence assays for $\alpha$-SMA and TGF $\beta 1$ on P3O}

The $\alpha$-SMA immunolabelling can be verified in the muscularis externa as well as in the muscularis mucosae. On the other hand, the TGFß1 labelling can be verified in regions of the muscularis externa, as well as in blood vessels and in portions of the villi, coinciding with parts of the muscularis mucosae (Fig. 5A-C). The co-localisation of TGF 1 and $\alpha$-SMA is observed in regions of the muscularis externa, as well as in regions of the muscularis mucosae; there is also exclusive immunolabelling for TGF 11 close to the smooth muscle inside the villi, as well as in blood vessels and haematopoietic cells (Fig. 5D).

\subsection{Immunohistochemical assays for PCNA}

The immunohistochemical assays for PCNA on P7 (6A-G) point to variations in the proliferative profile between the different compartments of the jejunum. On P7, scattered immunolabelling for PCNA is verified, indicating the low proliferative activity (Fig. 6D). Already in the Lieberkühn crypts, a large number of PCNA-positive cells can be verified (Fig. 6E), as well as in the outer longitudinal smooth muscle (Fig. 6F). Conversely, in the inner circular smooth muscle, there is scattered immunolabelling (Fig. 6G). On P30, immunohistochemical assays for PCNA (Fig. $6 \mathrm{H}-\mathrm{O}$ ) indicate an increase in the proliferative profile of the jejunum. In the muscularis externa, it is possible to observe a large number of PCNA-positive cells (Fig. 6L), which is also observed in the villi (Fig. 6M) and in the Lieberkühn crypts (Fig. 6N). An increase in the percentage of PCNA-immunolabelled cells between P7 and P30 was observed within Lieberkühn crypts (47.3 \pm 6 to $57 \pm 9)$, muscularis externa $(13.5 \pm 4$ to $24.7 \pm 6)$ and villi $(11.3 \pm 5$ for $41.6 \pm 6)$. Of the three compartments analysed, the highest increase in the percentage of PCNA-positive cells was observed in the villi (Fig. 6O) ( $p=6.9 \mathrm{E}-24$, ANOVA).

\section{Discussion}

The telocytes were detected in the jejunum of adult rats by means of electron microscopy. These were initially observed in the lamina propria, below the epithelial layer of the intestinal crypts and between the smooth muscle cells of the muscularis mucosae (Cretoiu et al. 2012). In the present study, telocytes can be verified by their morphological characteristics which are visible in light microscopy (long and thin cytoplasmic projections and cylindrical cell bodies) in the developing jejunum. They are present in the submucosa, as well as in the centre of the villi around the lacteals in P7. The presence of telocytes in the developing jejunum in gerbils was corroborated by immunofluorescence assays for CD34, which is the main marker for telocytes (Corradi et al. 2013). Telocytes are CD34-positive fibroblast-like cells that escape the morphology of blood vessels and haematopoietic cells, which are also CD34-positive; we verified such cells mainly inside the villi. Immunofluorescence assays for $\alpha$-SMA indicate that on P7, the muscularis externa is already developed, but the presence of muscularis mucosae is incipient. The later development of this layer of musculature was also verified in rats, in which the muscularis mucosae can be discriminated only on P8 (Kammeraad 1942; Eşrefoğlu and Çetin 2017). Furthermore, the co-localisation of CD34 with TGF $\beta 1$ was observed in the telocytes found in the villi of the developing jejunum. TGF $\beta 1$, in addition to several other functions, acts in the process of smooth muscle differentiation (Hong et al. 2004; Guo and Chen 2012). Previous in vitro studies have shown that telocytes secrete TGF $\beta 1$ (Sanches et al., 2016, 2017a), which may indicate that these cells could play an auxiliary role in the development of smooth muscle in the jejunum.

The smooth muscle layers of the intestine have mesenchymal origin and also act in the development of the tissue architecture of the intestine, generating compressive forces which are necessary for intestinal folding (Wells and Spence, 2014); thus, in view of the presence of interspersed telocytes in the muscularis mucosae (Cretoiu et al. 2012), it can be assumed that the telocytes can act in the tissue organisation of the intestine. Such a role was proposed for myocardial (Bani 2016) and prostatic telocytes (Sanches et al., 2017a). Later on in the jejunum development, on P30, we observed the expansion of $\alpha$-SMA immunolabelling, which indicates the expansion of the muscularis externa itself; it is also possible to discriminate the immunolabelling for $\alpha$-SMA in muscularis mucosae, which becomes well defined, thus indicating the development of this musculature. In the muscularis externa, it is possible to discriminate its two constituent components, the outer longitudinal smooth muscle and the inner circular smooth muscle. As for muscularis mucosae, it can be verified that it extends inside the Plicae circularis, reaching the interior of the villi.

On P30, CD34 immunolabelling is interspersed in the muscularis externa and is also present in blood vessels. Likewise, the c-Kit immunolabelling is verified in portions of the muscularis externa. The colocalisation of $\mathrm{CD} 34$ with c-Kit is observed in regions of the muscularis externa, in addition to dispersed blood vessels; however, it is possible to observe the existence of regions which are exclusively CD34- and c-Kitpositive. CD34 immunolabelling typical of telocytes (elongated sections) is verified in the central region of the villi and c-Kit immunolabelling appears scattered. No co-localisation of CD34 with c-Kit occurs in the villi; such evidence indicates that the telocytes remain present in the villi, in which the smooth muscle develops later. In the muscularis externa that develops earlier, telocytes (CD34-positive, or CD34 + c-Kit-positive) coexist with exclusively c-Kit-positive cells, which may be ICCs, whose main marker is c-Kit (Komuro and Zhou 1996). It was hypothesised that CD34-positive fibroblast-like stromal cells would be progenitor cells from a large number of cells (Sidney et al., 2014). In view of this, telocytes could be progenitor cells of ICCs, since they have markers for CD34 and c-Kit, a profile also found in precursor cells of ICCs in organotypic cultures (Lôrincz et al. 2008).

Weaning in the Mongolian gerbil occurs around the 30th postnatal day (Norris and Adams, 1972), whereas the same occurs earlier in the rat, on around the 21st day (Sengupta, 2013). The intestine reaches maturation with weaning; therefore, alterations in gene expression, and the presence of paracrine factors and cytokines are linked to this period (Schaeffer et al., 2000; Rakoff-Nahoum, 2015). In our study, on P7, we observed a great proliferative activity in the crypts of Lieberkühn, as well as in the outer longitudinal smooth muscle, whereas in the villi and the inner circular smooth muscle, only scattered PCNA immunolabelling is seen, which indicates a low proliferative activity. On P30, at the end of weaning, in both layers of the muscularis externa, it is possible to observe an increase in the number of PCNA-positive cells, which is also verified in the villi and the crypts of Lieberkühn. From the 
compartments analysed, the villi had the greatest increase in the number of PCNA-positive cells, indicating the greatest proliferative activity in this compartment. Such data corroborate the later development of muscularis mucosae compared to muscularis externa, as well as of the villi themselves compared to the other histological compartments of the jejunum. On P30, the jejunum still demonstrates a high proliferative profile in all of its compartments, indicating later maturation, which may reflect the longer gestational period and later weaning in the Mongolian gerbil compared to other laboratory rodents. In conclusion, even if it does not have to indicate the exact time of onset of telocytes in the jejunum, our results demonstrate these cells are present throughout the postnatal development of this organ in the Mongolian gerbil, exist in the vicinity of smooth muscles, are TGF $\beta 1$-positive and could be implicated in the development of these muscles. Moreover, telocytes exist together with ICCs and, in view of the dynamics of CD34 and c-kit expression throughout jejunum postnatal development, telocytes could constitute the progenitor cells of ICCs.

\section{Acknowledgements}

We thank to Luiz Roberto Falleiros Jr. and the all researchers of the Laboratory of Microscopy and Microanalysis for the technical support.

\section{Author contributions}

All authors (BCZ, BDAS, JSM, MFB, FCAS, CNB, MIZ, CMBB, SLF, RMG, PSLV and SRT) contributed to the design and interpretation of results and the revision of the manuscript. BDAS, BCZ and JSM performed the experiments. BDAS and BCZ wrote the manuscript, SRT and PSLV performed the final review of the manuscript.

\section{Competing interests}

The authors declared no potential conflicts of interest with respect to the research, authorship, and/or publication of this article.

\section{Funding}

The author(s) disclosed receipt of the following financial support for the research, authorship, and/or publication of this article: This study was financially supported by FAPESP (São Paulo Research Foundation); Number of contracts: 2013/15939-0, 2013/16443-9.

\section{Grant sponsors}

FAPESP (São Paulo Research Foundation); Number of contracts: 2013/15939-0, 2013/16443-9.

\section{References}

Aleksandrovych, V., Pasternak, A., Basta, P., Sajewicz, M., Walocha, J.A., Gil, K., 2017 Telocytes: facts, speculations and myths. Folia Med. Cracov. 57, 5-22.

Bani, D., 2016. Telocytes in cardiac tissue architecture and development. Adv. Exp. Med. Biol. 913, 127-137.

Bei, Y., Zhou, Q., Fu, S., Lu, D., Chen, P., Chen, Y., Wang, F., Xiao, J., 2015. Cardiac telocytes and fibroblasts in primary culture: different morphologies and immunophenotypes. PLoS One 10 (2), e0115991.

Carmona, I.C., Bartolomé, M.J.L., Escribano, C.J., 2011. Identification of telocytes in the lamina propria of rat duodenum: transmission electron microscopy. J. Cell. Mol. Med. 15 (1), 26-30.

Corradi, L.S., Jesus, M.M., Fochi, R.A., Vilamaior, P.S.L., Justulin, L.A., Góes, R.M., Felisbino, S.L., Taboga, S.R., 2013. Structural and ultrastructural evidence for telocytes in prostate stroma. J. Cell. Mol. Med. 17 (3), 398-406.

Creţoiu, S.M., Creţoiu, D., Popescu, L.M., 2012. Human myometrium - the ultrastructural 3D network of telocytes. J. Cell. Mol. Med. 16 (11), 2844-2849.

Cretoiu, D., Cretoiu, S.M., Simionescu, A.A., Popescu, L.M., 2012. Telocytes, a distinct type of cell among the stromal cells present in the lamina propria of jejunum. Histol. Histopathol. 27 (8), 1067-1078.

Cummins, A.G., Steele, T.W., Labrooy, J.T., Shearman, D.J., 1988. Maturation of the rat small intestine at weaning: changes in epithelial cell kinetics, bacterial flora, and mucosal immune activity. Gut 29 (12), 1672-1679.

Díaz-Flores, L., Gutiérrez, R., Pino García, M., González, M., Díaz-Flores, L., Francisco Madrid, J., 2016. Telocytes as a source of progenitor cells in regeneration and repair through granulation tissue. Curr. Stem Cell Res. Ther. 11 (5), 395-403.

Drumm, B.T., Koh, S.D., Andersson, K.-E., Ward, S.M., 2014. Calcium signalling in Cajallike interstitial cells of the lower urinary tract. Nat. Rev. Urol. 1411, 555-564.

Eşrefoğlu, M., Çetin, A., 2017. Development of small and large intestine. Bezmialem Science 4, 36-40.

Faussone-Pellegrini, M.S., Bani, D., 2010. Relationships between telocytes and cardiomyocytes during pre- and post-natal life. J. Cell. Mol. Med. 14 (5), 1061-1063.

Fochi, R.A., Santos, F.C.A., Goes, R.M., Taboga, S.R., 2013. Progesterone as a morphological regulatory factor of the male and female gerbil prostate. Int. J. Exp. Pathol. 94 (6), 373-386.

Guo, X., Chen, S.Y., 2012. Transforming growth factor- $\beta$ and smooth muscle differentiation. World J. Biol. Chem. 3 (3), 41-52.

Hayashi, N., Sugimura, Y., Kawamura, J., Donjacour, A.A., Cunha, G.R., 1991. Morphological and functional heterogeneity in the rat prostatic gland. Biol. Reprod. 45 (2), 308-321.

Hong, J.H., Song, C., Shin, Y., Kim, H., Cho, S.P., Kim, W.J., Ahn, H., 2004. Estrogen induction of smooth muscle differentiation of human prostatic stromal cells is mediated by transforming growth factor-beta. J. Urol. 171 (5), 1965-1969.

Iino, S., Nojyo, Y., 2009. Immunohistochemical demonstration of c-Kit-negative fibroblast-like cells in murine gastrointestinal musculature. Arch. Histol. Cytol. 72 (2), 107-115.

Kammeraad, A., 1942. The development of the gastro-intestinal tract of the rat. I. Histogenesis of the epithelium of the stomach, small intestine and pancreas. J. Morphol. 70, 323-351.

Kang, Y., Zhu, Z., Zheng, Y., Wan, W., Manole, C.G., Zhanga, Q., 2015. Skin telocytes versus fibroblasts: two distinct dermal cell populations. J. Cell. Mol. Med. 19 (11) $2530-2539$.

Komuro, T., Zhou, D.S., 1996. Anti-c-kit protein immunoreactive cells corresponding to the interstitial cells of Cajal in the guinea-pig small intestine. J. Auton. Nerv. Syst. 61 (2), 169-174.

Kuehn, R.E., Zucker, I., 1968. Reproductive behavior of the Mongolian gerbil (Meriones unguiculatus). J. Comp. Physiol. Psychol. 66 (3, Pt.1), 747-752.

Li, X., Lu, J., Wang, Y., Huo, X., Li, Z., Zhang, S., Li, C., Guo, M., Du, X., Chen, Z., 2016. Establishment and characterization of a newly established diabetic gerbil line. PLoS One 11 (7), e0159420.

Liang, J., Wu, Y.-L., Chen, B.-J., Zhang, W., Tanaka, Y., Sugiyama, H., 2013. The C-kit receptor-mediated signal transduction and tumor-related diseases. Int. J. Biol. Sci. 9 (5), 435-443.

Lima, R.F., Rodriguez, D.A., Campos, M.S., Biancardi, M.F., Santos, I.F., Oliveira, W.D., Cavasin, G.M., Marques, M.R., Taboga, S.R., Santos, F.C., 2015. Bisphenol-a promotes antiproliferative effects during neonatal prostate development in male and female gerbils. Reprod. Toxicol. 58, 238-245.

Lőrincz, A., Redelman, D., Horváth, V.J., Bardsley, M.R., Chen, H., Ördög, T., 2008. Progenitors of interstitial cells of Cajal in the postnatal murine stomach. Gastroenterology 134 (4), 1083-1093.

Loskota, W.J., Lomax, P., Rich, S.T., 1974. The gerbil as a model for the study of the epilepsies. Seizure patterns and ontogenesis. Epilepsia 15 (1), 109-119.

Matyja, A., Gil, K., Pasternak, A., Sztefko, K., Gajda, M., Tomaszewski, K.A., Matyja, M., Walocha, J.A., Kulig, J., Thor, P., 2013. Telocytes: new insight into the pathogenesis of gallstone disease. J. Cell. Mol. Med. 17 (6), 734-742.

Mou, Y., Wang, Y., Li, J., Lü, S., Duan, C., Du, Z., Yang, G., Chen, W., Zhao, S., Zhou, J., Wang, C., 2013. Immunohistochemical characterization and functional identification of mammary gland telocytes in the self-assembly of reconstituted breast cancer tissue in vitro. J. Cell. Mol. Med. 17 (1), 65-75.

Nicolescu, M.I., Bucur, A., Dinca, O., Rusu, M.C., Popescu, L.M., 2011. Telocytes in parotid glands. Anat Rec (Hoboken) 295 (3), 378-385.

Nicolescu, M.I., Popescu, L.M., 2012. Telocytes in the interstitium of human exocrine pancreas: ultrastructural evidence. Pancreas 41 (6), 949-956.

Norris, M.L., Adams, C.E., 1972. Mortality from birth to weaning in the Mongolian gerbil, Meriones unguiculatus. Lab. Anim. 6 (1), 49-53.

Pasternak, A., Szura, M., Gil, K., Matyja, A., 2016. Interstitial cells of Cajal (ICC). Folia Morphol. (Warsz) 75, 281-286.

Pasternak, A., Gajda, M., Gil, K., Matyja, A., Tomaszewski, K.A., Walocha, J.A., Kulig, J., Thor, P., 2012. Evidence of interstitial Cajal-like cells in human gallbladder. Folia Histochem. Cytobiol. 50 (4), 581-585.

Popescu, L.M., Faussone-Pellegrini, M.-S., 2010. Telocytes - a case of serendipity: the winding from Interstitial Cells of Cajal (ICC), via Interstitial Cajal-Like Cells (ICLC) to Telocytes. J. Cell. Mol. Med. 14 (4), 729-740.

Qi, G., Lin, M., Xu, M., Manole, G.G., Wang, X., Zhua, T., 2012. Telocytes in the human kidney cortex. J. Cell. Mol. Med. 16 (12), 3116-3122.

Rakoff-Nahoum, S., Kong, Y., Kleinstein, S.H., Subramanian, S., Ahern, P.P., Gordon, J.I., Medzhitov, R., 2015. Analysis of gene-environment interactions in postnatal development of the mammalian intestine. PNAS 112 (7), 1929-1936.

Ramón, Y., Cajal, S., 1911. Histologie du Systeme Nerveux de L'Homme et des Vertebres. Vol. 2 A. Maloine, Paris.

Salyards, G.W., Blas-Machado, U., Mishra, S., Harvey, S.B., Butler, A.M., 2013. Spontaneous Osteoblastic Osteosarcoma in a Mongolian Gerbil (Meriones unguiculatus). Comp Med. 63 (1), 62-66.

Sanches, B.D.A., Biancardi, M.F., Santos, F.C.A., Góes, R.M., Vilamaior, P.S.L., Taboga, S.R., 2014. Budding process during the organogenesis of the ventral prostatic lobe in mongolian gerbil. Microsc. Res. Tech. 77, 458-466.

Sanches B. D. A., Corradi L. S., Vilamaior P. S. L. and Taboga S. R. (2016) Paracrine signaling in the prostatic stroma: a novel role for the telocytes revealed in rodents 
ventral prostate. In: Xiangdong Wang; Dragos Cretoiu. (Org.). Advances in Experimental Medicine and Biology. 1ed.Singapore: Springer Singapore 1, 193-206.

Sanches, B.D.A., Maldarine, J.S., Zani, B.C., Tamarindo, G.H., Biancardi, M.F., Santos, F.C.A., Rahal, P., Goes, R.M., Felisbino, S.L., Vilamaior, P.S.L., Taboga, S.R., 2017a Telocytes play a key role in prostate tissue organisation during the gland morphogenesis. J. Cell. Mol. Med. 21 (12), 3309-3321.

Sanches, B.D.A., Maldarine, J.S., Zani, B.C., Biancardi, M.F., Santos, F.C.A., Góes, R.M., Vilamaior, P.S.L., Taboga, S.R., 2017b. Intrauterine exposure to 17ß-oestradiol (E2) impairs postnatal development in both female and male prostate in gerbil. Reprod. Toxicol. 73, 30-40.

Santos, F.C.A., Taboga, S.R., 2006. Female prostate: a review about the biological repercussions of this gland in humans and rodents. Anim. Reprod. 3 (1), 3-18.

Schaeffer, C., Diab-Assef, M., Plateroti, M., Laurent-Huck, F., Reimund, J.M., Kedinger, M., Foltzer-Jourdainne, C., 2000. Cytokine gene expression during postnatal small intestinal development: regulation by glucocorticoids. Gut 47 (2), 192-198.

Sengupta, P., 2013. The Laboratory rat: relating its age with human's. Int. J. Prev. Med. 4 (6), 624-630

Sidney, L.E., Branch, M.J., Dunphy, S.E., Dua, H.S., Hopkinson, A., 2014. Concise review: evidence for CD34 as a common marker for diverse progenitors. Stem Cells 32 (6), 1380-1389.

Spitzer, M.W., Semple, M.N., 1995. Neurons sensitive to interaural phase disparity in gerbil superior olive: diverse monaural and temporal response properties. J. Neurophysiol. 73 (4), 1668-1690.

Sugimura, Y., Cunha, G.R., Donjacour, A.A., 1986. Morphogenesis of ductal networks in the mouse prostate. Biol. Reprod. 34 (5), 961-971.

Sureda, E.A., Gidlund, C., Weström, B., Prykhodko, O., 2017. Induction of precocious intestinal maturation in T-cell deficient athymic neonatal rats. World $\mathrm{J}$. Gastroenterol. 23 (42), 7531-7540.

Taboga, S.R., Vilamaior, P.S.L., Góes, R.M., 2009. Androgenic and estrogenic modulation in the prostate: an approach in rodent experimental models with emphasis on structural biology. Arq Bras Endocrinol Metabol 53 (8), 946-955.

Thuneberg, L., 1982. Interstitial cells of Cajal: intestinal pacemaker cells? Adv. Anat. Embryol. Cell Biol. 71, 1-130.

Wells, J.M., Spence, J.R., 2014. How to make an intestine. Development 141, 752-760. Xiao, J., Bei, Y., 2016. Decoding telocytes. Adv. Exp. Med. Biol. 913, 23-39.

Xiao, J., Wang, F., Liu, Z., Yang, C., 2013. Telocytes in liver: electron microscopic and immunofluorescent evidence. J. Cell. Mol. Med. 17 (12), 1537-1542.

Zheng, Y., Bai, C.E., Wang, X., 2012. Potential significance of telocytes in the pathogenesis of lung diseases. Expert Rev. Respir Med. 6 (1), 45-49.

Zheng, Y., Cretoiu, D., Yan, G., Cretoiu, S.M., Popescu, L.M., Wang, X., 2014. Comparative proteomic analysis of human lung telocytes with fibroblastos. J. Cell. Mol. Med. 18 (4), 568-589. 